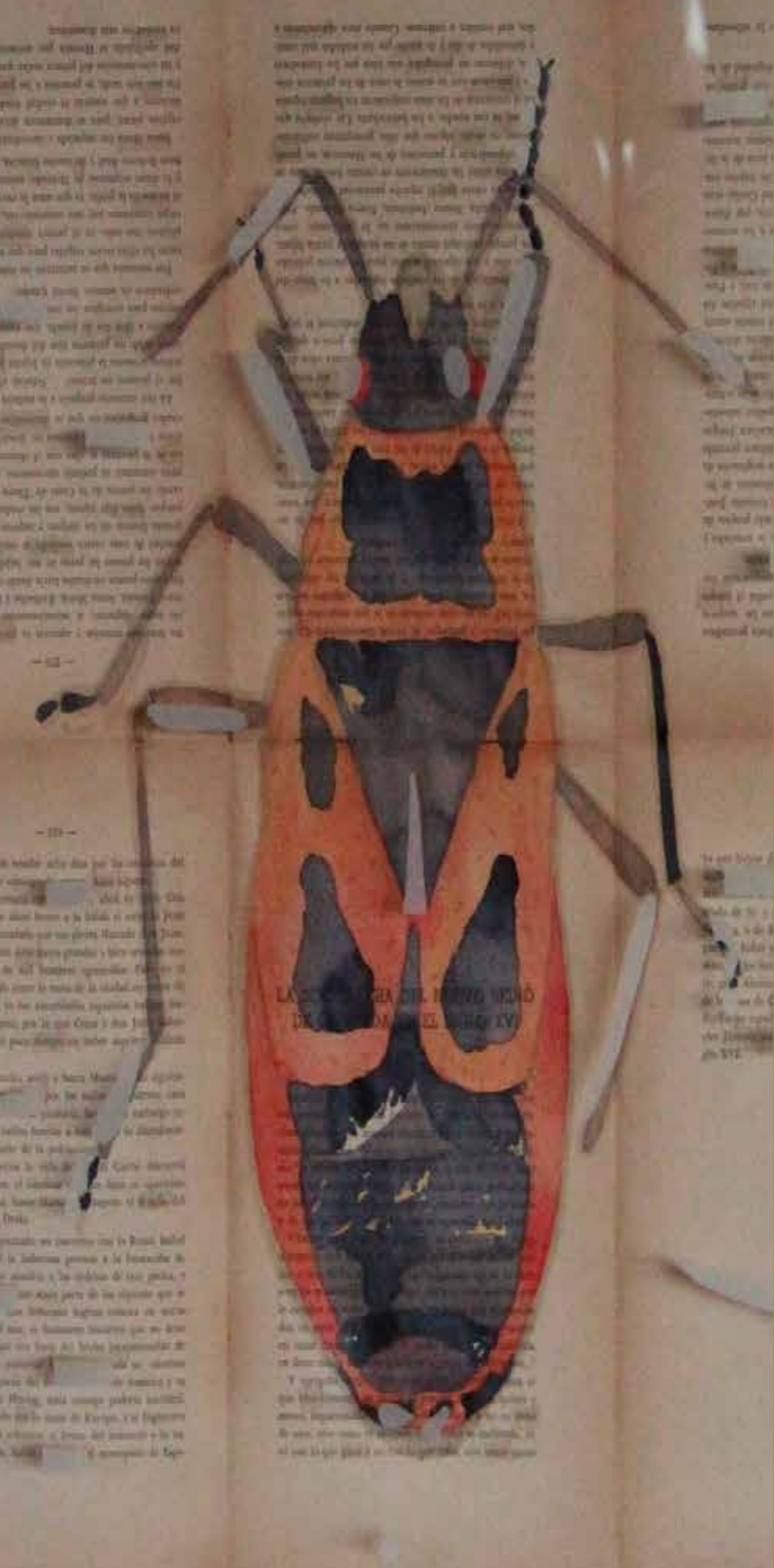

María Emma Reyes-Cuervo

Psicóloga candidata PHD, docente investigadora

Grupo de investigación en Capacidades

Humanas Salud e Inclusión

Escuela Colombiana de Rehabilitación, Bogotá, Colombia. mereyes@ecr.edu.co

\title{
Juanita Bejarano-Celis
}

Psicóloga, docente investigadora

Grupo de investigación en Capacidades Humanas Salud e Inclusión

Escuela Colombiana de Rehabilitación, Bogotá, Colombia. jebejarano@ecr.edu.co

\section{Tina Samper \\ Discapacidad Sensorial}

"Palabras Pintadas" Acuarela 


\section{Bibliometric study of the journal "Rehabilitation Psychology", from 2006 to 2012}

Fecha de recepción: 25 de marzo de 2013 - Fecha de aprobación: 19 de mayo de 2013

\section{RESUMEN}

Este estudio presenta un análisis bibliométrico de la revista Rehabilitation Psychology con el objetivo de identificar la evolución y las características de la producción del conocimiento en esta área de la psicología durante los últimos seis años, se propone retroalimentar a una comunidad científica que trabaja en diversidad e inclusión con respecto al estado de desarrollo del campo de la psicología de la rehabilitación. Se analizaron 318 artículos correspondientes a la totalidad de documentos publicados en formato electrónico entre los años 2006 y 2012. Los resultados muestran indicadores bibliométricos que incluyen indicadores métricos de productividad por países, instituciones, profesiones y autor (de acuerdo con la ley de lotka), así como una categorización rigurosa del contenido temático de la revista. Los resultados son analizados a la luz de la generación y aplicación del conocimiento especializado en el contexto local, así como los discursos vigentes de discapacidad. Finalmente el análisis permite resaltar la necesidad de promover la organización y difusión del conocimiento producido en el campo de la psicología de la rehabilitación en Colombia dentro de un marco para la inclusión social.

PALABRAS CLAVE

Psicología de la rehabilitación, bibliometría, CIF

\section{ABSTRACT}

This study presents a bibliometric analysis of the Rehabilitation Psychology review, with the objective of identifying the evolution and the characteristics of the production of knowledge in this area of the psychology during the last six years, the purpose is to feedback the scientific community that works in diversity and inclusion with respect to the state development of this field of study of Psychology Rehabilitation. 318 items corresponding to all papers published in electronic format were analyzed in the years between 2006 and 2012. The results showed a bibliometric indicator which included metrics of productivity for countries, institutions, professions and authors (according to the Lotka law), the same way a rigorous categorization of the thematically content of the review. The results are analyzed through the light of the generation and application of specialize knowledge in a local context, as well as diverge disability speeches. Finally highlight the need to promote the systematization and diffusion of knowledge produced in the field of psychological rehabilitation in Colombia in a framework of inclusion.

KEY WORDS

rehabilitation psychology, bibliometrics, ICF 
Salud la hace Florez-Alarcón (1999), quien presenta la trayectoria de esta área en la mayoría de países occidentales. Otro trabajo del mismo autor muestra un panorama de este campo en Colombia en el cual destaca la incursión de la psicología en el ámbito de la salud en la década de los años 80 que, como lo señala "condujo a la consolidación y desarrollo extenso de una psicología clínica de la salud, con énfasis en actividades de prevención secundaria y terciaria" (Florez-Alarcón, 2006, p. 689). Esta tendencia que permanece en los años 9o, dejó durante esa década la evidencia en la consolidación de asociaciones y la realización de Congresos Colombianos de Psicología de la Salud sin que aún se expresen énfasis en torno a temas relacionados directamente con la rehabilitación.

El interés por la producción científica en general, tiene su raíz en la necesidad de conocer con cierta precisión el estado y evolución de las diferentes áreas del conocimiento. La relevancia de la evidencia recapitulativa se fundamenta en la necesidad de consolidar la información del estado actual de la investigación sobre un tema determinado, que contribuya al mejoramiento de la comunicación científica y al desarrollo puntual y confiable de las profesiones y sus especialidades (Brugueras y Oramas, 2008; Fernández-Ríos y Buela-Casal, 2009; UNESCO, 1983). Este tipo de investigaciones son pertinentes dado que el conocimiento adquiere valor cuando se difunde y se da a conocer a la comunidad científica en general (Bordons y Zuleta, 1999).

La bibliometría, que como parte de la cienciometría, busca aportar información acerca de la actividad científica, ha permitido durante los últimos años avanzar en análisis cuantitativos de la producción científica, y aportar información importante sobre la calidad y el desarrollo de las disciplinas, áreas y temáticas particulares. De acuerdo con González-Pereira, Guerrero-Bote y Moya-Anegón (2010) la nueva investigación bibliométrica está dirigida a considerar no sólo el número de citaciones recibidas por un agente científico, sino también su importancia e influencia. En tal sentido, las nuevas métricas de la ciencia apuntan a dar cuenta no sólo de la cantidad de citaciones recibidas sino una combinación entre cantidad y calidad de la producción científica.

En psicología, los abordajes bibliométricos han dado cuenta del crecimiento y desarrollo de la disciplina, y han aportado al conocimiento en psicología clínica y de la salud como campos más próximos a la psicología de la rehabilitación. A continuación se reseñan algunos de los trabajos que anteceden a este estudio y dan cuenta de abordajes bibliométricos en la psicología en el contexto Ccolombiano.

Guerrero y Jaraba (2011) en un análisis bibliométrico de revistas relacionadas con la producción científica Colombiana (1949-2008), presentan el crecimiento de la producción en tres periodos específicos: a) entre 1969 y 1991 con una productividad baja, b) entre 1992 y 1996 con un aumento importante de los artículos publicados (5 a 20 artículos en un periodo cuatro veces más corto que el anterior), y c) entre 1997 y 2009 con un incremento que representa una tasa de crecimiento cercana al $17 \%$. Al observar el tipo de especialidades que se han mantenido en el periodo analizado, se visibiliza entre otras, la psicología clínica y de la salud, siendo esta especialidad la que cuenta con un número mayor de artículos en el periodo de 1997 a $2009(24 \%)$, mientras que durante la década comprendida entre 1998 
y 2008 obtuvo la segunda tasa de crecimiento (43\%). No obstante a propósito de la producción en psicología clínica, Rey, Martínez, y Guerrero (2009) analizaron la baja producción científica relacionada con el diagnóstico, la evaluación, el tratamiento y la prevención de trastornos diferentes a los de estado de ánimo particularmente al de ansiedad.

El análisis bibliométrico reportado por Quevedo-Blasto y LópezLópez (2010) con base en las revistas web of science (WoS) de psicología entre 2008 y 2009, que incluyó 448 trabajos, reportó el predominio de psicología social $(18.75 \%)$, seguidos por psicología clínica (16.74\%) y psicología educativa (11.16\%); cabe señalar que, aunque en menor porcentaje, también se reportan publicaciones en psicología de la rehabilitación (1.34\%). En cuanto a la producción por autor se hace evidente la ausencia de publicaciones con más de seis autores y de procedencia de países diferentes al de la revista. Con respecto al origen geográfico de las publicaciones, un análisis previo pero más amplio fue el realizado por Navarrete-Cortés, Chaichio-Moreno, Quevedo-Blasco y Ríos (2009), quienes revisaron la producción científica en psicología, a partir de 470 revistas con un total de 108.741 documentos, entre artículos originales y de revisión. Su estudio incluyó todos los países registrados en la Web of Science (WoS) y los resultados arrojaron que los países con mayor número de publicaciones son Estados Unidos (53.32\%) e Inglaterra (9.98\%). Los países con la tasa de mayor producción en psicología son Estados Unidos, Nueva Zelanda, Canadá, Países bajos y Australia, mientras que los que menos publican son India, Argentina, Polonia y Chile. Con respecto a los países de América Latina, se observó que Brasil es uno de los países que más publica junto con México. Un aspecto interesante que resaltan los autores en la investigación es que en la producción científica influye el tamaño demográfico y la renta per cápita, lo que se demuestra en la distribución por continentes, es decir, que la mayor producción científica está dada en mayor medida en Europa y América del Norte.

En lo relativo a los indicadores bibliométricos se observa la necesidad de estudiar índices de autor (autores más o menos productivos según número de artículos publicados), número de documentos disponibles por temática, distribución de documentos por países, metodologías utilizadas, temáticas de interés e idiomas de la publicación entre otros(Quevedo-Blasco, Ariza y Raya, 2012; Ariza y Granados, 2012), todo lo cual ha permitido sintetizar líneas de investigación llevar a cabo análisis de impacto académico y de transferencia del conocimiento principalmente.

En tanto los artículos científicos son considerados fuentes primarias que evidencian el estado del conocimiento sobre un tema, se constituyen en unidades de análisis fundamentales en torno al desarrollo del conocimiento en un área determinada. Este es el caso de la psicología de la rehabilitación y su publicación en la revista especializadas de la APA: Rehabilitation Psychology, que desde el año 2006 mostró un giro editorial importante en términos de la incorporación conceptual de la Clasificación Internacional del Funcionamiento, la Discapacidad y la Salud, CIF; situación que marcó la decisión metodológica de selección de las unidades a incluir en este análisis, especialmente si se tiene en cuenta que interesa explorar la producción científica y su proyección en el corto plazo especialmente en cuanto al contenido.
La revista Rehabilitation Psychology es el medio de publicación más importante de esta área de conocimiento en idioma inglés editada en Estados Unidos y proyectada como un espacio para el avance disciplinar y profesional en psicología de la rehabilitación. De acuerdo con la editorial de Wegener (2012) el aumento de la producción en la psicología de la rehabilitación ha sido estable durante los últimos cinco años. El alcance de la revista incluye un abordaje científico y tecnológico amplio desde la perspectiva de la psicología a la multiplicidad de variables biológicas, psicológicas, sociales, ambientales y políticas que están involucradas en el funcionamiento de las personas en situación de discapacidad o enfermedad crónica. Teniendo en cuenta la limitada información disponible en castellano de esta área de especialidad se ha decidido analizar la producción presentada en esta revista.

La relevancia de la Rehabilitation Psychology se soporta además en sus criterios de revisión de trabajos, según lo cual el aporte al campo de conocimiento, el rigor científico y la claridad de la escritura son aspectos centrales. Sin duda el aspecto más importante a considerar es el aporte a la mejor comprensión de los problemas que le competen a la psicología de la rehabilitación, especialmente lo que tiene que ver con una mejor intervención a los aspectos psicológicos en el contexto de la discapacidad y la enfermedad crónica. Esta investigación no pretendió determinar la calidad de la revista analizada, en cambio sí, de valorar y describir el estado de la producción del conocimiento en psicología de la rehabilitación a través de un análisis de la productividad y el contenido evidente en los artículos publicados en la revista durante un período reciente. El impacto social de este estudio radicó en la necesidad de impulsar esta área de la psicología en el contexto local, establecer evidencia para la construcción de herramientas que permitan la toma de decisiones en ámbitos políticos y la promoción de la salud en respuesta a las necesidades de las personas en condición de discapacidad o con enfermedad crónica.

Este estudio tuvo como propósito analizar la productividad científica en psicología de la rehabilitación a partir algunos indicadores métricos de productividad: por institución, por país de contacto, por número de autores y profesión, autores más productivos y contenido de suma importancia, de la revista Rehabilitation Psychology durante los años 2006 a 2012. Se espera que estos elementos sienten las bases para proyectar el campo del conocimiento en psicología de la rehabilitación a nivel local a partir del desarrollo de este campo evidenciado en una revista especializada de la APA.

\section{MÉTODO}

\section{Unidad de Análisis}

Las unidades de análisis del estudio corresponden a los artículos publicados en la Rehabilitation Psychology entre 2006 y 2012. Se analizaron 318 artículos entre los que se cuentan reportes de investigación, artículos de revisión y presentación de resultados de validación de instrumentos (ver Tabla 1). Se descartaron para este análisis los editoriales, las reseñas de libros y las opiniones de autores. 


\begin{tabular}{|c|c|c|c|c|c|}
\hline \multicolumn{5}{|c|}{ Tipo de Artículo } \\
\hline Año & $\begin{array}{c}\text { Reporte } \\
\text { de Investigación }\end{array}$ & Revisión & Validación & otros & Total \\
\hline 2006 & 30 & 3 & 5 & 1 & 39 \\
\hline 2007 & 48 & 3 & 3 & 0 & 54 \\
\hline 2008 & 34 & 2 & 2 & 16 & 54 \\
\hline 2009 & 40 & 5 & 7 & 1 & 53 \\
\hline 2010 & 39 & 2 & 2 & 2 & 45 \\
\hline 2011 & 27 & 5 & 6 & 1 & 39 \\
\hline 2012 & 31 & 0 & 3 & 1 & 34 \\
\hline Totales & 249 & 20 & 28 & 22 & 318 \\
\hline
\end{tabular}

Tabla 1. Tipos y distribución de artículos por años incluidos en el estudio.

La Rehabilitation Psychology, tuvo su primer número en el año 1955, es la publicación de la división 22 de la Asociación Americana de Psicología con cuatro números anuales que para 2012 llegó al volumen 57 y se encuentra incorporada en 22 servicios de indexación y resúmenes entre los que se cuentan CurrentAbstracts, PsycINFO, MEDLINE, PubMed, SCOPUS y Social Sciences Citation Index. La revista publica resultados de investigación experimental y no experimental, evaluación de intervenciones específicas, abordajes históricos, asuntos relacionados con la política pública, desarrollos conceptuales con implicaciones en la práctica clínica, estudios de caso, reflexiones profesionales, entre otros.

De acuerdo con SCImago Journal \& Country Rank (SJR, 2013) la Rehabilitation Psychology publicada por la Asociación Americana de Psicología en Estados Unidos, se inscribe en el área de la medicina, en la categoría de psiquiatría y salud mental. Esta revista es revisada trimestralmente por pares y los artículos que publica tienen el aval de la División 22 de la APA (APA, 2014).Como se observa en la Tabla 2, el indicador SJR en los últimos cinco años ha ido aumentando para algunos factores y fluctuado para otros. Lo que indica que el número de enlaces que la revista recibe por medio de la citación ponderada de sus documentos, asociada con el número de documentos publicados en el año por cada publicación, incrementa (Torres-Salinas y Jiménez-Contreras, 2010).

\begin{tabular}{|c|c|c|c|c|c|}
$\begin{array}{c}\text { Año de } \\
\text { publicación }\end{array}$ & $\begin{array}{c}\text { Indicador } \\
\text { SJR* }\end{array}$ & $\begin{array}{c}\text { Citas / doc. } \\
\text { (2 a) ** }\end{array}$ & $\begin{array}{c}\text { Citaciones } \\
\mathbf{( 3} \text { a) }\end{array}$ & $\begin{array}{c}\text { Citas } \\
\text { ext/ doc. } \\
\mathbf{3} \text { a) }\end{array}$ & $\begin{array}{c}\text { Colaboradores } \\
\text { inter con más } \\
\text { de un país) }\end{array}$ \\
\hline 2006 & 0,508 & 0,889 & 150 & 0,964 & $2,5 \%$. \\
\hline 2007 & 0,527 & 1,222 & 189 & 1,188 & $22,22 \%$ \\
\hline 2008 & 0,592 & 1,269 & 228 & 1,278 & $41,07 \%$ \\
\hline 2009 & 0,469 & 1,273 & 209 & 1,054 & $30,19 \%$ \\
\hline 2010 & 0,682 & 1,982 & 314 & 1,613 & $22,22 \%$ \\
\hline 2011 & 0,082 & 2,082 & 304 & 1,675 & $9,76 \%$ \\
\hline * & $\begin{array}{l}\text { Indicador de prestigio científico basado en sistemas de ponderación de citas y cen- } \\
\text { tralidad de vectores propios para ser utilizados en redes de citación complejos y } \\
\text { heterogéneos tales como Scopus. } \\
\text { Cita por documento (2a) mide el impacto cientifico con una ventana de dos años de } \\
\text { un artículo promedio publicado en la revista. }\end{array}$ \\
\hline
\end{tabular}

Tabla 2. Indicadores de citación de la revista Rehabilitation Psychology de acuerdo con SCImago.
Para cada una de las variables se identificó y registró información específica en un instrumento tipo base de datos para reducir la información de cada artículo tal como se detalla a continuación:

Año. Se refiere al año en que se publicó cada artículo (entre 2006 $y$ 2012). Cada año corresponde a un volumen.

Institución. Se refiere a la distribución de los artículos según la institución a que pertenece el autor principal, la cual suele ser en cabeza de la cual se encuentra el estudio reportado.

País de contacto. Se refiere al país en donde se encuentra el autor principal, esta información corresponde a la dirección de contacto ofrecida por el artículo.

Número de Autores. Corresponde a la cantidad de autores que reporta el artículo.

Registro profesional de los autores. Consiste en la profesión y especialidad de al menos los tres primeros autores que reporta el artículo, que corresponden a las disciplinas representativas que lideran el estudio.

Palabras clave. Incluyen todas las palabras clave reportadas en cada uno de los artículos incluidos

\section{Procedimiento}

Los artículos fueron recuperados desde la página de la revista y la información fue reducida en el instrumento cargado en una base de datos en Excel. El análisis de datos se llevó a cabo con la misma herramienta, lo que permitió hacer las estadísticas descriptivas presentadas.

El instrumento fue validado por dos jueces expertos con el fin de establecer criterios sobre las variables y su relación con los indicadores métricos a considerar (Anastasi, 1968 citado en Escurra, 1988). De esta manera se definieron los indicadores de productividad y de contenido que se describen a continuación.

La productividad por institución, por país y por profesión consistió en determinar la distribución de la producción según cada una de estas variables. La productividad científica de la Rehabilitation Psychology en este estudio, según institución, país, autor y profesión se fundamentó en el conteo de la producción según sea el caso, sin considerar el consumo del mismo en términos de citación, es por esto que no se hablará aquí de índices de productividad propiamente dichos. A cambio de esto se analizaron indicadores de productividad asociados a autores, países, instituciones y profesiones más productivas en el campo de la psicología de la rehabilitación, así mismo lo concerniente a los contenidos temáticos más prominentes.

Específicamente en cuanto a los autores más productivos se hizo uso de la Ley de Lotka, según la cual los autores se distribuyen de acuerdo a su nivel de productividad, así: Pequeños productores, serán aquellos autores que tienen un solo trabajo; el índice de pro- 
ductividad es igual a cero. Como medianos productores se refiere a aquellos autores que tienen entre 2 y 9 trabajos; el índice de productividad es mayor de cero y menor de 1 . Finalmente como grandes productores se conocen aquellos que tienen 10 trabajos o más; el índice de productividad es igual o mayor de 1 (Araújo y Arencibia, 2002). La productividad neta se ha contabilizado como la suma de publicaciones realizadas y se ha considerado la proporción relativa entre pequeños, medianos y grandes productores de acuerdo con la ley de Lotka.

En relación con el contenido temático, se realizó una categorización rigurosa de las palabras clave en coherencia con la literatura teórica y las tendencias temáticas disponibles en los manuales más recientes (Quevedo-Blasco \& López-López, 2010; Frank y Elliot, 2002).

\section{RESULTADOS}

Los resultados que se detallan a continuación representan la productividad científica de la revista Rehabilitation Psychology de acuerdo con los indicadores establecidos dentro del procedimiento, esto es, productividad (incluyendo el índice de Lotka) y contenido temático predominante.

En la Tabla 3 se observa el número de artículos por año y la proporción correspondiente del tamaño muestral considerado. De acuerdo con esta tabla, el mayor número de artículos está concentrado en los años 2007 a 2009, lo que corresponde al 50.55\% del total, con una diferencia importante respecto del año 2006, y un decremento constante a partir de 2009 hasta el 2012.

\begin{tabular}{|c|c|c|}
\hline Año & N & Porcentaje \\
\hline 2006 & 39 & $12.26 \%$ \\
\hline 2007 & 54 & $16.98 \%$ \\
\hline 2008 & 54 & $16.98 \%$ \\
\hline 2009 & 53 & $16.67 \%$ \\
\hline 2010 & 45 & $14.15 \%$ \\
\hline 2011 & 39 & $12.26 \%$ \\
\hline 2012 & 34 & $10.69 \%$ \\
\hline
\end{tabular}

Tabla 3. Número y porcentaje de artículos por año incluidos en el estudio

\section{Instituciones y regiones más productivas}

En la Tabla 4 se identifican los número de artículos y porcentajes totales de la participación de las instituciones con mayor número de publicaciones. Como se observa, la Universidad de la Alabama junto con la Universidad de Texas son las instituciones que publican en mayor medida con un total de participación en 16 trabajos cada una, correspondientes al $10.06 \%$ del total de los artículos publicados por la revista en el período estudiado. Es de anotar que estas dos instituciones participan no sólo como instituciones principales sino en colaboración con otras. A este respecto se destacan además de la Universidad de Alabama, la de Washington y la del Estado de Wayne, que aparecen como instituciones participantes en colaboración con otras, actuando como institución principal (de contacto), también como segunda y tercera mencionada según la afiliación de los autores. No sobra resaltar el hecho de que todas las mencionadas son instituciones académicas que se encuentran ubicadas en Estados Unidos. En general, la institución de salud que reporta mayor número de publicaciones es el Centro de Rehabilitación Metodista, con 6 publicaciones, adicionalmente, no se observa colaboración de ésta con otras instituciones, y representa un $1.89 \%$ de los artículos revisados.

\begin{tabular}{|c|c|c|c|c|c|}
\hline Institución 1 & $\begin{array}{c}\text { Frecuencia } \\
\text { institución } \\
\text { principal }\end{array}$ & $\begin{array}{l}\text { Frecuencia } \\
\text { segunda } \\
\text { institución }\end{array}$ & $\begin{array}{l}\text { Frecuencia } \\
\text { tercera } \\
\text { institución }\end{array}$ & $\begin{array}{l}\text { Frecuencia } \\
\text { total }\end{array}$ & Porcentaje \\
\hline $\begin{array}{l}\text { University of Alabama at } \\
\text { Birmingham }\end{array}$ & 12 & 2 & 2 & $16^{*}$ & $5,03 \%$ \\
\hline Texas A\&M University & 10 & 6 & - & 16 & $5.03 \%$ \\
\hline University of Washington & 6 & 3 & 3 & $12^{*}$ & $3.77 \%$ \\
\hline $\begin{array}{l}\text { Johns Hopkins University } \\
\text { School of Medicine }\end{array}$ & 9 & 2 & - & 11 & $3.46 \%$ \\
\hline Wayne State University & 5 & 2 & 2 & $9^{*}$ & $2.83 \%$ \\
\hline University of Florida & 6 & 2 & - & 8 & $2.52 \%$ \\
\hline McMaster University & 8 & - & - & 8 & $2.52 \%$ \\
\hline $\begin{array}{l}\text { University of Washington } \\
\text { School of Medicine }\end{array}$ & - & 5 & - & 5 & $1.57 \%$ \\
\hline $\begin{array}{l}\text { University of } \\
\text { Wisconsin-Madison }\end{array}$ & - & 3 & 2 & 5 & $1.57 \%$ \\
\hline University of Michigan & 6 & - & - & 6 & $1.89 \%$ \\
\hline $\begin{array}{l}\text { Methodist Rehabilitation } \\
\text { Center }\end{array}$ & 6 & - & - & 6 & $1.89 \%$ \\
\hline Northwestern University & - & 6 & - & 6 & $1.89 \%$ \\
\hline Baylor College of Medicine & - & 3 & - & 3 & $0.94 \%$ \\
\hline $\begin{array}{l}\text { Rehabilitation Institute of } \\
\text { Michigan }\end{array}$ & - & 3 & - & 3 & $0.94 \%$ \\
\hline $\begin{array}{l}\text { University of } \\
\text { Saskatchewan }\end{array}$ & - & 2 & - & 2 & $0.63 \%$ \\
\hline University of Kansas & - & 2 & - & 2 & $0.63 \%$ \\
\hline Hurley Medical Center & - & 2 & - & 2 & $0.63 \%$ \\
\hline University of lowa & - & 2 & - & 2 & $0.63 \%$ \\
\hline $\begin{array}{l}\text { American Psychological } \\
\text { Association }\end{array}$ & - & 2 & - & 2 & $0.63 \%$ \\
\hline $\begin{array}{l}\text { University of Oklahoma } \\
\text { Health Sciences Center }\end{array}$ & - & 2 & - & 2 & $0.63 \%$ \\
\hline $\begin{array}{l}\text { Princess Margaret } \\
\text { Hospital }\end{array}$ & - & 2 & - & 2 & $0.63 \%$ \\
\hline University of South Florida & - & 2 & - & 2 & $0.63 \%$ \\
\hline Sheffield Health & - & 2 & - & 2 & $0.63 \%$ \\
\hline University of Dundee & - & - & 2 & 2 & $0.63 \%$ \\
\hline $\begin{array}{l}\text { University of Colorado } \\
\text { Denver }\end{array}$ & - & - & 2 & 2 & $0.63 \%$ \\
\hline Cleveland State University & - & - & 2 & 2 & $0.63 \%$ \\
\hline University of Pittsburgh & - & - & 2 & 2 & $0.63 \%$ \\
\hline University of Alberta & - & - & 2 & 2 & $0.63 \%$ \\
\hline
\end{tabular}

Tabla 4. Participación de las instituciones en las investigaciones según la afiliación reportada por los autores.

En la Tabla 5 se describe la distribución de los artículos por países. Se evidencia que Estados Unidos es el país con el mayor número de trabajos publicados (228), es decir, cerca del $72 \%$ del total, seguido de Canadá con un $8.81 \%$. Cabe destacar la enorme diferencia entre el primero y el segundo, así como el hecho de que países de habla hispana no reportan publicaciones en dicha revista. 


\begin{tabular}{|l|c|c|}
\multicolumn{1}{|c|}{ País } & Frecuencia & Porcentaje \\
\hline Estados Unidos & 228 & $71.70 \%$ \\
\hline Canadá & 28 & $8.81 \%$ \\
\hline Inglaterra & 22 & $6.92 \%$ \\
\hline Australia & 10 & $3.14 \%$ \\
\hline Alemania & 6 & $1.89 \%$ \\
\hline Holanda & 4 & $1.26 \%$ \\
\hline China & 3 & $0.94 \%$ \\
\hline Escocia & 3 & $0.94 \%$ \\
\hline Noruega & 2 & $0.63 \%$ \\
\hline Francia & 2 & $0.63 \%$ \\
\hline Nueva Zelanda & 2 & $0.63 \%$ \\
\hline España & 2 & $0.63 \%$ \\
\hline Israel & 2 & $0.63 \%$ \\
\hline Suiza & 1 & $0.31 \%$ \\
\hline Suecia & 1 & $0.31 \%$ \\
\hline Indiana & 1 & $0.31 \%$ \\
\hline Singapur & 1 & $0.31 \%$ \\
\hline
\end{tabular}

Tabla 5. Participación por país según autor principal.

Profesiones más productivas en psicología de la rehabilitación

Se describen hasta tres de las profesiones o sus especialidades reportadas en los artículos a fin de observar las relaciones entre profesiones en el área temática. En la tabla 6 se muestra que la mayoría de autores principales son psicólogos (cerca del 41\% sin contar la neuropsicología), seguido de los médicos alrededor del 26\% (sin contar las especialidades) y de los kinesiólogos y fisioterapeutas con un $5 \%$. Las especialidades que participan con mayor frecuencia son la psiquiatría, la neurología, la neuropsicología y la pediatría.

Es de anotar que si bien psicología, medicina y psiquiatría son registros profesionales con mayor participación en la autoría principal, también lo son en cuanto a participación en colaboración, lo que no ocurre con la kinesiología, la cual registra una participación importante en la autoría principal pero no así como segunda profesión aportante. Es importante resaltar en la autoría principal y colaboración otras áreas de la salud diferentes a la medicina, como son la kinesiología, la enfermería y la terapia ocupacional, mientras que otras áreas del conocimiento como son la educación y las ciencias sociales (sin contar la psicología) aparecen tímidamente en el panorama; entre ambas suman un total de 9 autores.

Si se tiene en cuenta las profesiones o especialidades reportadas en segunda y tercera instancia dentro de los artículos, se destaca la contribución de la medicina, la psicología y la psiquiatría nuevamente y se destacar la participación de la neurología y la educación. La anestesiología, la ingeniería biomecánica, la estadística y la ortopedia son registros profesionales que aparecen exclusivamente como colaboraciones y no como líderes de investigación, lo cual sugiere desde ya el papel de estas áreas como apoyo a la comprensión de los objetos de estudio.

\begin{tabular}{|c|c|c|c|c|c|}
\hline $\begin{array}{l}\text { Profesión / } \\
\text { especialidad }\end{array}$ & $\begin{array}{l}\text { Profesión } \\
\text { autor } \\
\text { principal }\end{array}$ & $\begin{array}{l}\text { Segunda } \\
\text { profesión }\end{array}$ & $\begin{array}{c}\text { Tercera } \\
\text { profesión }\end{array}$ & $\begin{array}{c}\text { Frecuencia } \\
\text { total }\end{array}$ & $\begin{array}{l}\text { Porcenta- } \\
\text { je autoría } \\
\text { principal }\end{array}$ \\
\hline Psicología & 132 & 19 & 5 & 156 & $41.51 \%$ \\
\hline Medicina & 83 & 29 & 9 & 121 & $26.10 \%$ \\
\hline Psiquiatría & 15 & 14 & 1 & 30 & $4.72 \%$ \\
\hline $\begin{array}{l}\text { Kinesiologia/ } \\
\text { fisioterapia }\end{array}$ & 16 & 1 & - & 17 & $5.03 \%$ \\
\hline Neurología & 3 & 11 & 2 & 16 & $0.94 \%$ \\
\hline Neuropsicología & 9 & 1 & 1 & 11 & $2.83 \%$ \\
\hline Pediatría & 5 & 1 & 3 & 9 & $1.57 \%$ \\
\hline Enfermería & 5 & 2 & - & 7 & $1.57 \%$ \\
\hline $\begin{array}{c}\text { Terapia } \\
\text { ocupacional }\end{array}$ & 4 & 1 & 1 & 6 & $1.26 \%$ \\
\hline $\begin{array}{l}\text { Educación/ } \\
\text { Pedagogía }\end{array}$ & 2 & 4 & - & 6 & $0.63 \%$ \\
\hline Epidemiologia & 1 & 2 & 1 & 4 & $0.31 \%$ \\
\hline $\begin{array}{l}\text { Ciencias } \\
\text { Sociales }\end{array}$ & 2 & 1 & - & 3 & $0.63 \%$ \\
\hline Biomedicina & 1 & - & - & 1 & $0.31 \%$ \\
\hline Anestesiología & - & 2 & 1 & 3 & - \\
\hline $\begin{array}{l}\text { Ingeniería } \\
\text { Biomecánica }\end{array}$ & - & 1 & 1 & 2 & - \\
\hline Estadística & - & 2 & & 2 & - \\
\hline Ortopedia & - & 1 & 1 & 2 & - \\
\hline Sin dato & 40 & & & & $12.58 \%$ \\
\hline TOTAL & 318 & & & & \\
\hline
\end{tabular}

Tabla 6. Participación por registro profesional de acuerdo con su lugar de mención en los artículos.

\section{Productividad por autor}

La coautoría es una constante en los artículos publicados en la Rehabilitation Psychology. La tabla 7 presenta el número de autores por artículo, observándose que la mayoría de artículos son escritos por mínimo tres autores (casi el 26\%), seguido de cuatro y dos autores en coautoría. En menor medida escriben en un artículo solamente un autor o más de 7 .

La tabla 8 presenta la distribución en las tres categorías de acuerdo al número de trabajos producidos por los autores durante los años 2006 a 2012 en la Rehabilitation Psychology. Este análisis consideró hasta los primeros seis autores reportados en los artículos. Se evidencia que en mayor medida hay pequeños productores con un porcentaje cercano al $67 \%$; mientras que hay un porcentaje menor de pequeños productores casi un $33 \%$ sin que se encontraran grandes productores.

\section{Contenido temático}

En total se contabilizaron 1381 palabras clave que fueron categorizadas como se describe a continuación.

Diagnóstico psicológico/psiquiátrico y síntomas asociados. Incluye las palabras clave referentes a trastornos psiquiátricos definidos por el DSM IV o síntomas que se relacionan con los mismos. Por ejemplo se desatacan en esta categoría "depresión" y trastornos 


\begin{tabular}{|c|c|}
\hline No autores & Porcentaje \\
\hline 3 & $25.79 \%$ \\
\hline 4 & $19.50 \%$ \\
\hline 2 & $16.35 \%$ \\
\hline 5 & $14.78 \%$ \\
\hline 6 & $9.43 \%$ \\
\hline 1 & $5.66 \%$ \\
\hline 7 & $4.09 \%$ \\
\hline 8 & $1.89 \%$ \\
\hline 9 & $1.26 \%$ \\
\hline 10 & $0.31 \%$ \\
\hline 13 & $0.31 \%$ \\
\hline 22 & $0.31 \%$ \\
\hline
\end{tabular}

Tabla 7. Número de autores que contribuyen en cada artículo.

\begin{tabular}{|l|c|c|}
\hline Categorias Ley de Lotka & Frecuencia & Porcentaje \\
\hline Pequeños productores & 819 & $67.13 \%$ \\
\hline Medianos productores & 401 & $32.86 \%$ \\
\hline Grandes productores & 0 & 0 \\
\hline
\end{tabular}

Tabla 8. Nivel de productividad por autor.

de "ansiedad", particularmente el "Estrés Postraumático" así como síntomas asociados ("síntomas") y síndromes asociados a la "fatiga" y al "burnout".

Diagnóstico médico /condición de salud física. Se refiere a la patología médica o lesión de base que a menudo son objeto de análisis de los artículos. Por ejemplo se destacan como es la "lesión de médula espinal", "trauma craneoencefálico", "ECV" y "esclerosis múltiple".

Variables psicológicas. Se incluyen bajo esta categoría una diversidad de palabras clave que suponen aspectos inherentes al ser humano y que han sido consideradas en las investigaciones como aspecto de interés, a menudo estas son variables objeto de estudio o son consideradas como factores de riesgo o protectores. Se incluyen palabras clave como "afrontamiento", "autoeficacia", "autoestima", "ajustamiento" "resiliencia” "satisfacción con la vida”, "esperanza”, "autonomía”, "espiritualidad” entre otras.

Conceptos derivados de la CIF. Comprende palabras clave que expresan directamente "discapacidad" o palabras compuestas con esta misma (por lo general referentes a tipos de discapacidad) también se destacan "limitaciones en la actividad" así como "función", "funcionamiento" y "limitación". Estas palabras claves son agrupadas en una misma categoría como referentes léxicos de la perspectiva propuesta por la CIF.

Soporte social. Son aquellas palabras clave que se refieren al apoyo y funcionamiento de redes sociales, especialmente estas pala- bras incluyen lo relacionado con el papel de cuidadores y familiares como soporte social. Por ejemplo se tienen palabras clave como "cuidado", "cuidadores", "apoyo social”, "integración comunitaria”, "rol de la familia", entre otras.

Contexto sociocultural. Esta categoría estrechamente relacionada con la anterior busca sacar a un lado las palabras clave que refieren un contexto más amplio que el directamente asociado a condiciones de salud; contempla entonces situaciones socioculturales como "empleo", sectores como "educación" "ambiente" "política pública" "discriminación”, entre otras.

Condiciones adversas y trauma. Existe un número de palabras clave que refieren condiciones ambientales y personales tales como "trauma" o condiciones traumáticas tales como "catástrofes" "violencia" o "abuso".

Rehabilitación. Directamente se refiere a aquellas palabras clave que expresan "rehabilitación", se tiene entonces la misma palabra u otras compuestas como "psicología de rehabilitación", "rehabilitación cognitiva" o "resultados de la rehabilitación"

Grupos poblacionales (étnicos, etáreos, género). A menudo las palabras clave se refieren a los grupos poblacionales objeto de atención en los estudios, así, bajo esta categoría se incluyen palabras clave como "veteranos", "género", "niños", "mujeres", "adolescencia" o "envejecimiento", entre otras.

Procesos psicológicos. Corresponde a las funciones psicológicas como son la cognición, la motivación y la emoción. Se incluyen bajo esta categoría directamente el uso de éstas como palabra clave"

Evaluación. Bajo esta categoría se incluyen las palabras clave directamente relacionadas con "evaluación", así como palabras compuestas que la incorporan, por ejemplo "evaluación ecológica”.

Intervención. Contempla aspectos relacionados con la implementación de alguna estrategia terapéutica. Se incluyen palabras clave como "terapia de grupo", "telesalud" "resultados", entre otras

Metodología. Abarca aquellas palabras clave relacionadas con aspectos propios de la investigación. Se incluyen palabras como: "análisis factorial", "análisis cualitativo", "ecuaciones estructurales”, entre otros.

Es de anotar que un análisis preliminar de frecuencias de palabras clave se observa la depresión como una de las palabras mayormente reportada, así como discapacidad y rehabilitación, ocurre algo similar con palabras de corte médico como lesión en la médula espinal y lesión cerebral.

En la tabla 9 se muestra la distribución por frecuencia de las palabras clave incluidas en los artículos y categorizadas de acuerdo con los criterios descritos arriba. Así mismo, se ha avanzado en una re-categorización mediante la agrupación de las categorías previstas. De esta forma pueden observarse nuevas categorías emergentes en el análisis previsto de manera que existe un grupo temático de 
tipo clínico que propone el abordaje de diagnósticos y síntomas físicos y psicológicos en donde la intervención sobre variables psicológicas puede ser preponderante. Otro grupo temático deja ver una vertiente social del abordaje en donde los criterios de la Clasificación Internacional del Funcionamiento y la Discapacidad juegan un papel importante. Finalmente está la dimensión metodológica de la investigación que aporta una importante vertiente temática para la psicología de la rehabilitación. Como se observa en la tabla 9, existiría un grupo temático "residual" que deja enunciadas categorías claves y posiblemente prometedoras.

\section{DISCUSIÓN}

De acuerdo con la editorial de Wegener (2012) el aumento de la producción en la psicología de la rehabilitación ha sido estable durante los últimos cinco años, lo cual se corrobora en el estudio presentado, en donde además se evidencia una tendencia temática fuerte, así como una forma de producción que también manifiestan una preferencia, o si se quiere una forma de ser de la producción científica del tema en la revista seleccionada. Sin duda como lo señala Quevedo-Blasto y López-López (2010), en la producción científica influye el tamaño demográfico y la renta per cápita, y seguramente el volumen de producción de Estados Unidos está influenciado por este hecho; no obstante para el caso de la Rehabilitation Psychology no debe desestimarse el hecho de que la revista es editada y publicada en ese país.

La productividad por país e institución, que está fuertemente ligada por las condiciones geográficas a que ambos aspectos hacen referencia, da cuenta de la amplia producción de literatura científica en psicología de la rehabilitación en Estados Unidos, especialmente en la University of Alabama at Birmingham en donde se encuentran, vinculados al departamento de Psicología, tres iniciativas asociadas directamente al área de rehabilitación así: 1) The Taub Therapy Clinic, que en colaboración con el Sistema de Salud de la UAB se dedica al estudio y atención de pacientes que han sufrido Enfermedad Cerebro Vascular y Trauma Cráneo Encefálico. 2) The Center for Research on Applied Gerontology con foco de atención sobre la población mayor y los campos de investigación que se derivan de allí como son la independencia, la calidad de vida y la productividad, entre otros tópicos. 3) el Civitan International Research Center, que tiene como uno de sus temas centrales de interés la discapacidad en niños, y tal como lo expresan en su página web tiene como misión "mejorar el bienestar y la calidad de vida de las personas y las familias afectadas por discapacidades intelectuales y de desarrollo".

Se trata pues de un referente institucional clave en materia de psicología de la rehabilitación, en tanto proponen una perspectiva interdisciplinaria e interinstitucional de investigación en el campo de la rehabilitación de la discapacidad asociada a accidentes, envejecimiento y desarrollo. Estos hallazgos en este centro académico explican la alta productividad de la institución así como su capacidad de colaboración en los temas de interés de la revista, y dejan ver desde ya las áreas de desarrollo en psicología de la rehabilitación que están impulsando.

La psicología de la rehabilitación es un campo que si bien tiene una trayectoria importante en la historia de la división 22 de la APA, es aún fructífero y al parecer poco fortalecido en cuanto a la presencia de grandes productores, al menos al interior de esta revista. Tendría entonces que revisarse más ampliamente la productividad de los autores con mayor número de publicaciones a fin de determinar con mayor precisión si estamos ante una baja productividad por autor en el área de Psicología de la Rehabilitación, quizás resulte arriesgado este tipo de afirmaciones si se tiene en cuenta que este indicador de productividad está limitado a una única revista, que aún cuando podría considerarse una de las más importantes en la materia, es solamente una en la cual los autores han podido decidir exponer sus trabajos.

Tal y como se observa en los resultados hay una importante contribución a los temas de psicología de la rehabilitación desde las áreas de la salud; así la medicina (y sus especialidades), la enfermería y profesiones de la rehabilitación, específicamente la fisioterapia y terapia ocupacional tienen una participación importante en la producción científica revisada, lo que se decanta en el abordaje

\begin{tabular}{|l|c|c|}
\hline \multicolumn{1}{|c|}{ Categoría temática } & N de palabras clave & Porcentaje \\
\hline Diagnóstico Médico / Condición de Salud Física & 248 & $18,0 \%$ \\
\hline Variables Psicológicas & 235 & $17,0 \%$ \\
\hline Intervención & 112 & $8,1 \%$ \\
\hline Diagnóstico psic./psiqu. y síntomas asociados. & 111 & $8,0 \%$ \\
\hline Conceptos derivados de CIF & 102 & $7,4 \%$ \\
\hline Soporte Social & 102 & $7,4 \%$ \\
\hline Contexto Sociocultural & 82 & $5,9 \%$ \\
\hline Metodología & 84 & $6,1 \%$ \\
\hline Grupos Poblacionales & 61 & 4,4 \\
\hline Rehabilitación & 60 & 4,3 \\
\hline Evaluación & 41 & 3,0 \\
\hline Condiciones Adversas y Trauma & 30 & 2,2 \\
\hline Procesos Psicológicos & 25 & 1,8 \\
\hline otras (no clasificadas) & 88 & $6,1 \%$ \\
\hline
\end{tabular}

Tabla 9. Categorización de palabras clave

66 REV. COL. REH || Bogotá, Colombia || Volumen 12 || Páginas 58 - 69 || ISSN 1692 - 1879 
interdisciplinar de los temas relacionados con discapacidad y enfermedad crónica.

La presencia de áreas como la fisioterapia y la terapia ocupacional se explica especialmente por la relación entre movilidad y bienestar psicológico, que comprende uno de los tópicos sobresalientes en cuanto a abordaje de ECV principalmente. En general es esperable la interrelación de la psicología con áreas de la salud específicas como psiquiatría, neurología, pediatría o enfermería, especialmente dado los tópicos concernientes con enfermedad crónica y accidentes, no obstante es interesante el alto volumen de artículos dirigidos por profesionales de esta área, quizás también explicable por la pertenencia de la revista a la categoría de Medicina. Esto, sin embargo expresa también el aporte importante que está llamada a hacer la psicología a los temas de salud de la población.

Por su parte algunas disciplinas provenientes de las ciencias sociales, entre las que se cuentan la sociología, la pedagogía y la educación, tienen una menor participación en las publicaciones revisadas, no obstante aportan los temas que se abordan desde la psicología de la rehabilitación.

Si a lo anterior se suma el hecho de evidenciar en los resultados una importante referencia a través de las palabras clave, a los aspectos concernientes con variables sociales tales como redes de soporte social y contexto social y cultural de la discapacidad, sin duda se trata de una especialidad de la psicología que logra permear tanto ámbitos del sector salud, como lo correspondiente a educación y comunidad.

De esta manera puede entenderse que la psicología de la rehabilitación favorece a otras áreas específicas de la psicología, aportando a los diferentes ámbitos en los que se desenvuelve el sujeto, que a su vez es un ser político. La presencia de variables psicológicas propias de la práctica clínica y de la salud como son "afrontamiento", "autoeficacia", "autoestima", "ajustamiento" "resiliencia" "satisfacción con la vida", "esperanza”, "autonomía”, "espiritualidad”, pero también aspectos asociados a la práctica educativa y comunitaria de la psicología como son "psicosocial" "funcionamiento familiar" "cuidadores" o "apoyo social", son evidencia de la participación y aporte que debe hacer la psicología de la rehabilitación a favor de la calidad de vida y la inclusión de personas con discapacidad, enfermedad crónica o quienes han enfrentado traumas que los han dejado en condición de exclusión.

Un análisis más profundo del contenido temático deja ver unas tendencias de investigación en psicología de la rehabilitación que ha promovido la vinculación de las variables psicológicas con las condiciones de salud física y psicológica de las personas en la búsqueda de encontrar caminos adecuados de intervención. Lo anterior marca lo que son las raíces del la psicología de la salud, con un desarrollo tan prominente en Colombia (Florez-Alarcón, 1999, 2006). No obstante más allá de esta tendencia marcada también en la rehabilitation psychology, pueden vislumbrarse en nuestro análisis temas emergentes que han venido fortaleciéndose en los últimos años, así, las variables sociales y culturales parecen cobrar mayor relevancia en los abordajes en torno a la rehabilitación. En este sentido, se destacan el papel de los contextos sociales educativos, de empleabilidad, de inclusión, de apoyo familiar y cuidado entre otros.

Otras temáticas que aparecen en el análisis de palabras clave dejan ver los campos que puede ser promisorios en Colombia, especialmente si se tiene en cuenta el proceso de recuperación tras una guerra de más de 50 años; esto es, lo relacionado con trauma y condiciones adversas, en donde las cuestiones relacionadas con evaluación e intervención, y rehabilitación de grupos particulares cobran relevancia.

Una representación del análisis expuesto entorno a las temáticas de la psicología de la rehabilitación se representa en la figura 1, la cual contempla tres áreas específicas de la psicología que plantea la producción de la revista Rehabilitation Psychology durante los últimos seis años, como son la psicología clínica y de la salud, la psicología social y comunitaria, y la psicología de la educación, que a su vez pueden contribuir de manera bidireccional a la psicología de la rehabilitación que como hemos visto se complementa interdisciplinarmente con las profesiones de la salud. Todo lo anterior bajo un marco de Derechos de los grupos poblacionales más vulnerables: veteranos, mujeres, niños y adolescentes (mayormente definidos por palabras claves en las publicaciones de la revista), que contribuya al bienestar del sujeto en condición de discapacidad o con enfermedad crónica.

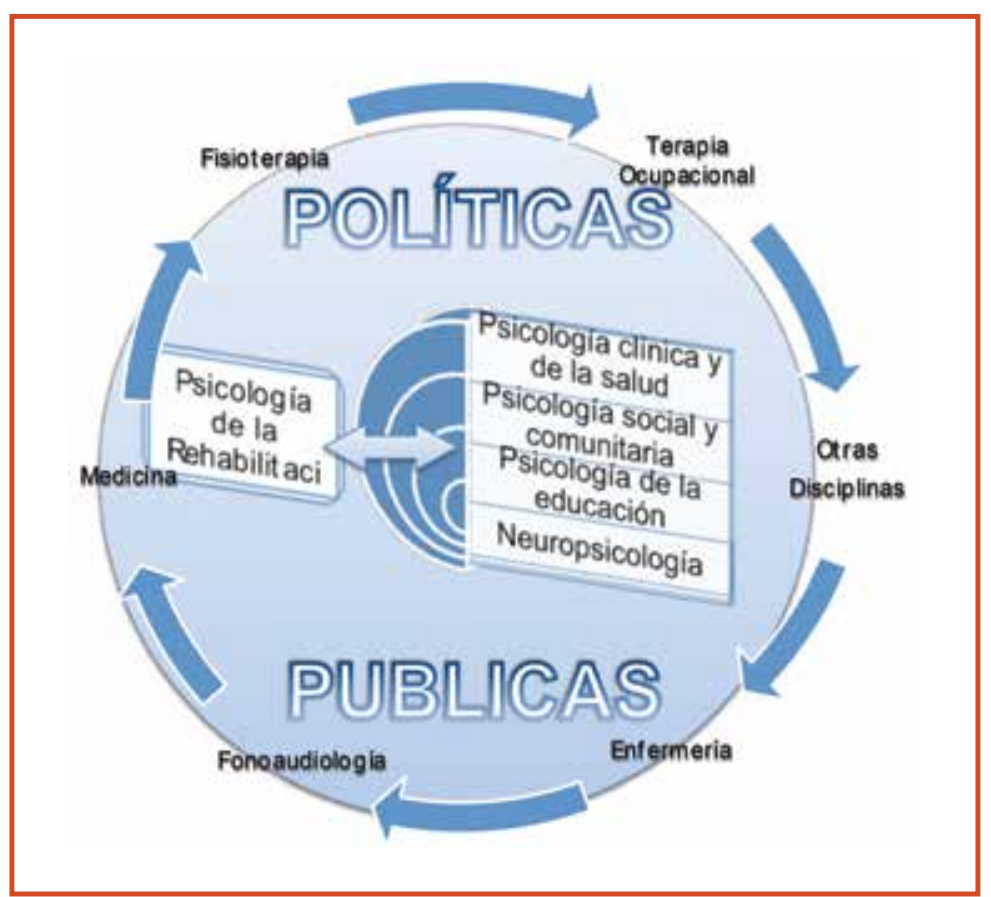

Figura 1. Prospectiva de la Psicología de la Rehabilitación en el contexto local a partir de la participación de las profesiones y el contenido de suma importancia en la investigación reportada en la revista Rehabilitation Psychology entre 2006 y 2012 .

En este marco, hablar del respeto de los derechos a las personas en condición de discapacidad y el de sus familias, implica trabajar sobre la rehabilitación integral (Brogna, 2006), lo que conlleva a replantear el trabajo desde una sola disciplina o profesión, de manera que se requiere de varios frentes que hagan su apuesta desde sus 
7 objetos de estudio, para observar al ser humano de manera holística y así contribuir en su bienestar y proceso de inclusión en todos los ámbitos. Para ello, se amerita una reflexión sobre los perfiles, funciones y comprensión de los objetos de estudio desde las diferentes profesiones. Al mismo tiempo se contempla la atención integral a los usuarios y a sus familias, desde un enfoque de derechos teniendo presentes las especificidades idiosincráticas de cada escenario.

Esto requiere de trabajos más exhaustos que promueva análisis más complejos que permitan analizar la incidencia de varios factores sobre la variable discapacidad. Al respecto, en una investigación se evaluaron las tendencias investigativas comprendidas entre el 2000 y el 2009, en la educación superior en el Suroccidente Colombiano. Se observó una predominancia epistemológica empírico-inductiva, como segunda tendencia se destaca la introspectiva vivencial, y se percibe la ausencia de trabajos de corte racional deductivo. Por otra parte, la mayoría de las investigaciones revisadas por los autores, centradas en personas en condición de discapacidad con limitaciones físicas, adoptaban para su análisis el modelo biopsicosocial. También se destaca que los análisis tienden a ser descriptivos, por lo que es necesario buscar análisis explicativos que permitan la formulación de modelos teóricos para seguir avanzando en la ciencia. Asimismo, se plantea descentralizar los temas relacionados con las personas en condición de discapacidad, es decir, pasar de un nivel médico a uno participativo en las diferentes esferas sociales como son la educación, el trabajo, la comunidad, entre otros (Vanegas-García y Ortíz-Cabrera, 2013).

Es pues de suma importancia promover la investigación y la difusión del conocimiento en los ámbitos de la psicología clínica y de la salud, de la psicología social y comunitaria y la psicología de la educación, en el que se visibilicen las estrategias de abordaje bajo un marco para la inclusión. Aunque en el contexto local se ha trabajado el tema, en psicología aún falta fortalecerlo (Colegio Colombiano de psicología, auxiliar de dirección, mayo 15 de 2012). Por lo tanto, para trabajos futuros en psicología de la rehabilitación se deben promover la identificación de los campos temáticos y áreas de desarrollo específicas en el contexto local de cara al desarrollo de este campo en Colombia.

\section{Referencia:}

Las referencias a otras obras son una parte muy importante en la literatura científica;
ya que estas permiten conocer más sobre los autores y
mantener vivas sus voces dentro del texto.

Agudelo, D., Buela-Casal, G., \& Bretón- López, J. (2003). Análisis bibliométrico de las revistas de Psicología Clínica editadas en castellano. Psicothema, 15, 507-516. Disponible en www.psicothema.com.

American Psychological Association (2012). About division 22. Recuperado el 18 de junio de 2012 de http://www.apadivisions.org/division-22/about/ index.aspx.

American psychological Association (2014). Rehabilitation psychology. Recuperado el 10 de febrero de 2014 de http://www.apadivisions.org/division-22/publications/index.aspx

Araújo, J.A., y Arencibia, R. (2002). informetría, bibliometría, y cienciometría: aspectos teóricos prácticos. ACIMED, 10, 5-6. Disponible en: http://bvs.sld.cu/revistas/aci/volio_4_02/acio40402.htm.

Ariza, T. \& Granados, M. (2012). Análisis Bibliométrico de las revistas Iberoamericanas más relevantes afines a la psicología clínica y salud del Journal Citation Reports (2011). Terapia psicológica, 30, 89-102. Disponible en http://pepsic.bvsalud.org/scielo.php?script=sci_arttext\&pi$\mathrm{d}=$ So120-05342007000300011\&lng=pt\&nrm=iso.

Bordons, M., \& Zuleta, A. (1999). Evaluación de la actividad científica a través de indicadores bibliométricos. Revista española de cardiología, 52, 79080o. Disponible en: http://www.revespcardiol.org/

Brogna, P. (2006). El nuevo paradigma de la discapacidad y el rol de los profesionales de la rehabilitación. Revista Electrónica. Cuadernos Esp. Disponible en: http://www.esp.ce.gov.br/

Brugueras, C \& Oramas, J. (2008). Síntesis de información de artículos de revisión. Acimed, 17, 1-11. Disponible en: http://bvs.sld.cu/revistas/aci/ vol17_2_08/acio7208.htm]

Carvajal-Castrillón, J., Henao, E., Claramónika Uribe, P., \& Giraldo, M. (2009). Caso Clínico/Artículo de Revisión: Rehabilitación cognitiva en un 
caso de alteraciones neuropsicológicas y funcio-finales por Traumatismo Craneoencefálico severo1. Revista Chilena de Neuropsicología, 4, 52-63. Disponible en 63. www.neurociencia.cl

Colegio Colombiano de psicología (Comunicación personal 15 de mayo de 2012).

Escurra, L. M. (1988). Cuantificación de la validez de contenido por criterio de jueces. Revista de Psicología, 6(1-2), 103-111.

Fernández-Ríos, L. \& Buela-Casal, G. (2009). Standards for the preparation and writing of Psychology review articles. International Journal of Clinical and Health Psychology, 9,329-334. Disponible en: http://www.aepc.es/ ijchp/articulos_pdf/ijchp-326.pdf

Florez-Alarcón, L. (1999). Origen y Evolución de la psicología de la salud en Colombia. Revista Colombiana de Psicología, edición especial, 125-126.

Florez-Alarcón, L. (2006). La Psicología de la Salud en Colombia. Universitas Psicologica, 5, 681-693. Disponible en: <http://revistas.javeriana.edu.co/ index.php/revPsycho/article/view/470

Frank, R., \& Elliott, T. R. (Eds.). (2002). Handbook of rehabilitation psychology. Washington, DC: American Psychological Association.

Galeano, L. M. (2009). La Neuropsicología en Colombia. Revista Neuropsicología, Neuropsiquiatría y Neurociencias, 9, 47-52.

González Pereira, B., Guerrero Bote, V. P., \& Moya Anegón, F. (2010). A New Approach to the Metric of Journals' Scientific Prestige: The SJR Indicator. Journal of Informetrics, 4, 379-391.

Guerrero, J. \& Jaraba, B. (2011). La producción científica de la psicología colombiana: un análisis bibliométrico de las revistas académicas, 1949-2008. Grupo de Estudios Sociales de la Ciencia, la Tecnología y la Medicina. Bogotá: Universidad Nacional de Colombia.

Mejía-Castrejón, J., Rojas-Castillo, E., Balarezo-Jaramillo,V., Núñez-Valencia, C., \& Carrillo-Navarrete, G. (2011). Avances hacia una psicología científica en oncología. Vol, 10, 150.155.

Navarrete-Cortés, J., Chaichio-Moreno, J.A., Quevedo-Blasco, R. \& Ríos, C. (2009). Análisis cuantitativo por países de la productividad en psicología de las revistas en la web of science, 26, 131-143.
Organización de las Naciones Unidas para la educación de la Ciencia y la cultura. .(UNESCO, 1983). Guía para la redacción de artículos científicos destinados a la publicación. (2ed). París. Recuperado el 1 de junio de 2012 de http://www3.uva.es/iuu/DownLoads/o6_Guia_UNESCO.pdf.

Quevedo-Blasco, R \& López-López, W. (2010). Análisis Bibliométrico de las Revistas Multidisciplinares de Psicología Recientemente Incorporadas en la Web of Science (2008-2009). Psicologia: Reflexão e Crítica, 23, 384-408.

Quevedo-Blasco, R., Ariza, T., \& Raya, L. (2012). Análisis de la producción de la psicología jurídica en España (1989-2010). Aula abierta, 40, 127-138.

Rey, C., Martínez, J. \& Guerrero, S. (2009). Tendencias de los artículos en psicología clinica en Iberoamerica. Terapia psicológica, 27, 61-77.

Rueda, M. B., \& Aguado, A. L. (2003). Estrategias de Afrontamiento y proceso de adaptación a la Lesión Medular. Madrid: Ministerio de Trabajo y Asuntos SocialesInstituto de Migraciones y Servicios Sociales (IMSERSO).

SCImago Journal \& Country Rank (SJR). (2013). Journal search. Rehabilitation psychology. Recuperado el 13 de abril de 2013 de http://www.scimagojr. com/journalsearch.php?q=14112\&tip=sid\&clean $=0$

Torres-Salinas, D. y Jiménez-Contreras, E. (2010). Introducción y estudio comparativo de los nuevos indicadores de citación sobre revistas científicas en Journal Citation Reports y Scopus. El Profesional de la Información, 19 (2), 201-207.

Vanegas García, J. H., \& Ortiz Cabrera, M. F. (2013). Tendencias investigativas en discapacidad en instituciones de educación superior del suroccidente Colombiano 2000-2009 (Doctoral dissertation). Recuperado el 15 de enero de 2014 de http://repositorio.autonoma.edu.co/jspui/bitstream/11182/301/1/ TENDENCIAS\%2OINVESTIGATIVAS\%20EN\%20DISCAPACIDAD $\% 20$ EN\%2OINSTITUCIONES\%2oDE\%20EDUCACI\%C3\%93N\%20SUPERIOR\%20DEL\%2OSUROCCIDENTE.pdf

Wegener, S.T. (2012). Opening Commentary: Rehabilitation Psychology, 2012-2017. Rehabilitation Psychology, 57 (1) 1-4.

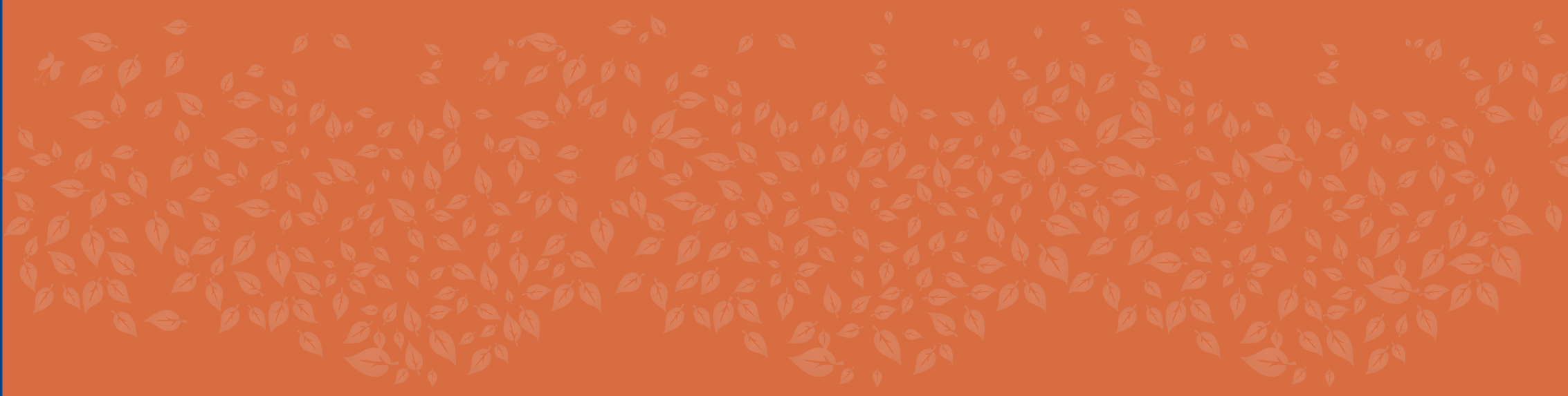

REV. COL. REH || Bogotá, Colombia || Volumen 12 || Páginas 58 - 69 || ISSN 1692 - 1879 